\title{
ON THE DISTRIBUTION OF THE ROOTS OF A POLYNOMIAL WITH INTEGRAL COEFFICIENTS
}

\author{
ROBERT BREUSCH
}

The following question has been raised by D. H. Lehmer ${ }^{1}$ in connection with prime number problems: "If $\epsilon$ is a positive quantity, to find a polynomial of the form $f(z) \equiv z^{r}+a_{1} z^{r-1}+\cdots+a_{r}$ where the $a$ 's are integers, such that the absolute value of the product of those roots of $f$ which lie outside the unit circle, lies between 1 and $1+\epsilon . "$ Lehmer calls this absolute value $\Omega(f)$. Since, for $f(z)=z^{2}-z-1$, $\Omega(f)=1.6 \cdots$, we may assume $\epsilon<1$, and therefore $a_{r}= \pm 1$. Then $\Omega(f)$ may be written in the form $\prod_{n=1}^{r}\left|z_{n}\right|^{\left[\left|z_{n}\right|\right]}$, where the $z_{n}$ are the roots of $f(z)$. It is shown in this paper that no nonreciprocal polynomial $f(z)$ exists with $\Omega(f)<1.179$. Lehmer himself gives an example of a reciprocal polynomial of degree 10 for which $\Omega(f)$ is less than this, namely $1.176 \cdots$; no nonreciprocal polynomial seems to be known for which $\Omega(f)<1.32$. Thus the present result is still far from being a complete answer to the question.

Let $f(z) \equiv z^{r}+a_{1} z^{r-1}+\cdots \pm 1$ be a nonreciprocal polynomial with integral coefficients, irreducible in the rational field. The proof makes use of the resultant $R(f, g)$ of $f(z)$ and the polynomial $g(z) \equiv \pm z^{r} f(1 / z)$ $=z^{r}+\cdots$. All the roots of $g(z)$ are of the form $z_{n}^{*} \equiv \bar{z}_{n}^{-1}$ where the $\bar{z}_{n}$ are the conjugates of the roots $z_{n}$ of $f(z)$. Since $f(z)$ and $g(z)$ are irreducible and different, have integral coefficients and highest coefficients $1,|R(f, g)| \equiv \prod_{n=1}^{r} \prod_{m=1}^{r}\left|z_{n}-z_{m}^{*}\right|$ must be not less than 1 .

$$
|R(f, g)| \equiv \prod_{n=1}^{r} \prod_{m>n}\left|z_{n}-z_{m}^{*}\right| \cdot\left|z_{m}-z_{n}^{*}\right| \cdot \prod_{n=1}^{r}\left|z_{n}-z_{n}^{*}\right| \equiv P_{1} \cdot P_{2}
$$

If $\Omega(f)=k$, it will be shown in Lemma 2 that $P_{1}<k^{2 r} \cdot r^{r}$, and in Lemma 3 that $P_{2} \leqq(4(k-1) / r)^{r}$.

Lemma 1. $P_{1} \equiv \prod_{n=1}^{r} \prod_{m>n}\left|z_{n}-z_{m}^{*}\right| \cdot\left|z_{m}-z_{n}^{*}\right|$ has the greatest value compatible with the two conditions (I) $\prod_{n=1}^{r}\left|z_{n}\right|=1$, (II) $\prod_{n=1}^{r}\left|z_{n}\right|^{\left[\left|z_{n}\right|\right]=k}(1<k<2)$ for $\left|z_{1}\right|=k,\left|z_{2}\right|=\cdots=\left|z_{r-1}\right|=1$, $\left|z_{r}\right|=1 / k$.

PRoof. For $n=1,2, \cdots, r$, call $b_{n}$ values of the $z_{n}$ for which $P_{1}$ takes its greatest value when restricted by conditions $I$ and II.

Presented to the Society, April 28, 1951; received by the editors January 2, 1951.

1 D. H. Lehmer, Factorization of certain cyclotomic functions, Ann. of Math. vol. 34 (1933) p. 476. 
Assume that at least two of the $b$ 's are absolutely greater than 1 , for example, $\left|b_{1}\right|>1,\left|b_{2}\right|>1 . P_{1}$ may be rewritten in the form

$$
\begin{gathered}
P_{1} \equiv \mid\left(z_{1}-\bar{z}_{2}^{-1}\right)\left(\bar{z}_{2}-z_{1}^{-1}\right) \cdot \prod_{n=3}^{r}\left(z_{1}-z_{n}^{*}\right)\left(\bar{z}_{n}-z_{1}^{-1}\right)\left(\bar{z}_{2}-z_{n}^{-1}\right)\left(z_{n}-\bar{z}_{2}^{-1}\right) \\
\cdot \prod_{n=3}^{r} \prod_{m>n}\left(z_{n}-z_{m}^{*}\right)\left(z_{m}-z_{n}^{*}\right) \mid .
\end{gathered}
$$

The expression within the absolute bars is now an analytic function of $z_{1}$ and $\bar{z}_{2}$ (but not of the remaining $z$ 's). Calling this expression $F\left(z_{1}, \bar{z}_{2}, z_{3}, \cdots, z_{r}\right)$, and introducing $A \equiv b_{1} \bar{b}_{2}$ (with $1<|A| \leqq k$ ), our assumption implies that $F\left(z, A / z, b_{3}, \ldots, b_{r}\right)$ which is analytic in $1 \leqq|z| \leqq|A|$ takes its largest value in this region for $z=b_{1}$. But this is impossible, for $b_{1}$ is by assumption an interior point of this region, because $1<\left|b_{1}\right|<|A|$.

Interchanging the $z_{n}$ with the $z_{n}^{*}$ does not change $P_{1}$; thus not two of the $\left|b_{n}^{*}\right|$ can be greater than 1 , that is, not two of the $\left|b_{n}\right|$ can be less than 1 .

Therefore (by proper arrangement of the subscripts) $P_{1}$ takes its greatest value for $z_{1}=k e^{i \theta_{1}}, z_{1}^{*}=(1 / k) e^{i \theta_{1}}, z_{r}=(1 / k) e^{i \theta_{r}}, z_{r}^{*}=k e^{i \theta_{r}}$, $z_{n}=e^{i \theta_{n}}, z_{n}^{*}=z_{n}(1<n<r)$.

LeMMA 2. $P_{1}<k^{2 r} \cdot r^{r}$.

Proof. Using the values found in Lemma 1 , we get, for $1<n<r$ '

$$
\begin{aligned}
\left|z_{1}-z_{n}^{*}\right| \cdot\left|z_{n}-z_{1}^{*}\right| & =\left|k e^{i \theta_{1}}-e^{i \theta_{n}}\right| \cdot \frac{1}{k} \cdot\left|k e^{i \theta_{n}}-e^{i \theta_{1}}\right| \\
& =\frac{1}{k}\left|z_{1}-z_{n}\right|^{2}
\end{aligned}
$$

(since $\left|k e^{i \theta_{n}}-e^{i \theta_{1}}\right|=\left|e^{i \theta_{n}}-k e^{i \theta_{1}}\right|$ ). Similarly, for $1<n<r$,

$$
\begin{gathered}
\left|z_{r}-z_{n}^{*}\right| \cdot\left|z_{n}-z_{r}^{*}\right|=k \cdot\left|z_{r}-z_{n}\right|^{2} \\
\left|z_{1}-z_{r}^{*}\right| \cdot\left|z_{r}-z_{1}^{*}\right|=\left|e^{i \theta_{1}}-e^{i \theta_{r}}\right|^{2}<\left|z_{1}-z_{r}\right|^{2} .
\end{gathered}
$$

Therefore

$$
\begin{gathered}
P_{1}<\left|z_{1}-z_{r}\right|^{2} \cdot \prod_{n=2}^{r-1}\left|z_{1}-z_{n}\right|^{2} \cdot\left|z_{r}-z_{n}\right|^{2} \cdot \prod_{n=2}^{r-1} \prod_{n<m<r}\left|z_{n}-z_{m}\right|^{2} \\
=\prod_{n=1}^{r} \prod_{m>n}\left|z_{n}-z_{m}\right|^{2} .
\end{gathered}
$$


The function

$$
\begin{aligned}
G(z) \equiv \frac{1}{z^{2 r}}\left(z-\frac{z_{1} z_{r}}{z}\right)^{2} & \cdot \prod_{n=2}^{r-1}\left(z-z_{n}\right)^{2} \cdot\left(\frac{z_{1} z_{r}}{z}-z_{n}\right)^{2} \\
& \cdot \prod_{n=2}^{r-1} \prod_{n<m<r}\left|z_{n}-z_{m}\right|^{2}
\end{aligned}
$$

is analytic for $1 \leqq|z| \leqq \infty$, and has therefore its greatest absolute value in this region for $|z|=1$. But for $|z|=1,|G(z)|$ is the absolute value of the discriminant of the polynomial whose $r$ roots $z, z_{1} z_{r} / z, z_{2}$, $\cdots, z_{r-1}$, all lie on the unit circle. This is known ${ }^{2}$ to be not greater than $r^{r}$. Therefore $|G(z)|<r^{r}$ for $|z|>1$, and $P_{1}<\left|z_{1}^{2 r}\right| \cdot\left|G\left(z_{1}\right)\right|$ $<k^{2 r} \cdot r^{r}$.

LEMMA 3. If the $z_{n}$ satisfy conditions I and II of Lemma 1, then $P_{2} \equiv \prod_{n=1}^{r}\left|z_{n}-z_{n}^{*}\right| \leqq(4(k-1) / r)^{r}$.

Proof. If $\left|z_{n}\right|=1+\epsilon_{n} \geqq 1$ for $n=1,2, \cdots, s$, and $\left|z_{n}^{*}\right|=\left|z_{n}\right|^{-1}$ $=1+\epsilon_{n}>1$ for $n=s+1, \cdots, r$, then $\left|z_{n}-z_{n}^{*}\right|=\left.|| z_{n}|-| z_{n}\right|^{-1} \mid=1$ $+\epsilon_{n}-1 /\left(1+\epsilon_{n}\right) \leqq 2 \epsilon_{n}$ for $n=1,2, \cdots, r$. Also, since $\prod_{n=1}^{s}\left(1+\epsilon_{n}\right)=k$, and $\prod_{n=s+1}^{r}\left(1+\epsilon_{n}\right)=k, 1+\sum_{n=1}^{s} \epsilon_{n} \leqq k, 1+\sum_{n=s+1}^{r} \epsilon_{n} \leqq k$, and $\sum_{n=1}^{r} \epsilon_{n}$ $\leqq 2(k-1)$. Therefore $P_{2} \leqq \prod_{n=1}^{r}\left(2 \epsilon_{n}\right) \leqq(4(k-1) / r)^{r}$.

THEOREM. If $f(z)$ is a nonreciprocal irreducible polynomial with integral coefficient and highest coefficient 1 , then $\Omega(f)=k>1.179$.

Proof. If $r$ is the degree of $f(z)$, the statement is obviously correct for $\left|a_{r}\right|>1$, since $k \geqq\left|a_{r}\right|$. Thus we may assume $\left|a_{r}\right|=1$. Then by Lemmas 2 and 3, $|R(f, g)| \equiv P_{1} P_{2}<k^{2 r} r^{r}(4(k-1) / r)^{r}=\left(4 k^{2}(k-1)\right)^{r}$. This must be not less than 1 ; therefore $4 k^{2}(k-1) \geqq 1, k>1.179$.

Amherst College

2 I. Schur, Ueber die Verteilung der Wurzeln bei gewissen algebraischen Gleichungen mit ganzzahligen Koeffizienten, Math. Zeit. vol. 1 (1918) p. 385. 